\title{
PAPER
}

\section{Abnormalities of cerebral perfusion in multiple sclerosis}

\author{
W Rashid, L M Parkes, G T Ingle, D T Chard, A T Toosy, D R Altmann, M R Symms, P S Tofts, \\ A J Thompson, D H Miller
}

J Neurol Neurosurg Psychiatry 2004;75:1288-1293. doi: 10.1136/jnnp.2003.026021

See end of article for authors' affiliations

Correspondence to:

Correspondence to:
Professor D H Miller, NMR

Research Unit, Department

of Neuroinflammation,

Institute of Neurology,

University College London,

Queen Square, London

WCIN 3BG, UK:

d.miller@ion.ucl.ac.uk

Received 15 August 2003

Revised 20 October 2003

Accepted

26 November 2003

\begin{abstract}
Background: Measuring perfusion provides a potential indication of metabolic activity in brain tissue. Studies in multiple sclerosis (MS) have identified areas of decreased perfusion in grey matter (GM) and white matter (WM), but the pattern in clinical subgroups is unclear.

Objectives: This study investigated perfusion changes in differing MS clinical subgroups on or off $\beta$ interferon therapy using a non-invasive MRI technique (continuous arterial spin labelling) to investigate whether different clinical MS subtypes displayed perfusion changes and whether this could give a further insight into the pathological mechanisms involved.

Methods: Sixty patients (21 relapsing remitting, 14 secondary progressive, 12 primary progressive, 13 benign) and 34 healthy controls were compared. Statistical parametric mapping (SPM '99) was used to investigate regional variations in perfusion in both GM and WM. Global WM perfusion was derived by segmenting WM from images using $T_{1}$ relaxation times.

Results: Regions of lower perfusion in predominantly GM were observed in the primary and secondary progressive cohorts, particularly in the thalamus. Increased WM perfusion was seen in relapsing remitting and secondary progressive cohorts.

Conclusions: Low GM perfusion could reflect decreased metabolism secondary to neuronal and axonal loss or dysfunction with a predilection for progressive forms of MS. Increased WM perfusion may indicate increased metabolic activity possibly due to increased cellularity and inflammation. Improved methodology and longitudinal studies may enable further investigation of regional and temporal changes, and their relationship with physical and cognitive impairment.
\end{abstract}

MS. In this present work, we estimated cerebral perfusion using continuous arterial spin labelling (CASL), an arterial spin labelling (ASL) technique that continuously labels arterial water in a proximal inversion plane. Image acquisition occurs in a distal area after a delay time to allow a high proportion of the labelled water to reach the sampling section. ${ }^{16}$ This method has the advantage of a higher signal to noise ratio compared with other ASL techniques. We obtained absolute measurements of perfusion in 60 MS subjects and 34 healthy volunteers in order to investigate: $(a)$ the potential of a non-invasive in vivo method to detect cerebral perfusion abnormalities in MS and $(b)$ regional patterns of perfusion abnormality in both GM and WM and in clinical MS subgroups. We hypothesised that decreased perfusion would, in reflecting neuronal and axonal loss, be associated with progressive and disabling forms of MS.

\section{METHODS \\ Subjects}

Using previously defined criteria, ${ }^{17} 60$ subjects (mean age 48.1 years, range 17-69, median EDSS 4; 21 male, 39 female) from four different subgroups of clinically definite $M^{18}$ (21 relapsing remitting, 14 secondary progressive, 13 benign and 12 primary progressive) were compared with 34 healthy controls (mean age 40.7 years, range 20-67; 15 male, 19 female). In addition, the relapsing remitting cohort was subdivided into those who were on $\beta$-interferon $(n=11$; median treatment 1.6 years (range 0.15-2.05)) and those not

Abbreviations: ASL, arterial spin labelling; CASL, continuous arterial spin labelling; EPI, echo planar imaging; FOV, field of view; GM, grey matter; MS, multiple sclerosis; MSFC, MS Functional Composite; PD, proton density; PET, positron emission tomography; SPECT, single photon emission computed tomography; SPM, statistical parametric mapping; TE, echo time; TR, repetition time; WM, white matter
GM and WM. ${ }^{13-15}$ Little work has been performed to investigate perfusion abnormalities in all the clinical subgroups of 
$(\mathrm{n}=10)$ (table 1$)$. No subject had a previous history of any other significant central nervous or systemic autoimmune conditions. Because of the potential effect on cerebral perfusion, subjects with a history of cerebrovascular disease, psychomotor depression, illicit drug use, poorly controlled hypertension, or ongoing medication with an immunosuppressant other than $\beta$-interferon were also excluded. Subjects were scanned at least 30 days after a relapse or steroid treatment.

All MS subjects underwent a neurological examination to evaluate their EDSS $^{19}$ and MS Functional Composite (MSFC) scores. ${ }^{20}$ The latter is a combined objective measure of upper and lower limb function and cognition. Written informed consent was obtained from all subjects and the study had approval by the ethics committee of the National Hospital for Neurology and Neurosurgery.

\section{Magnetic resonance imaging protocol}

All scans were acquired on a Signa 5.6 1.5 T system (General Electric, Milwaukee, USA). A CASL pulse sequence using gradient echo planar imaging (EPI) was performed. ${ }^{16}$ The scanning parameters were: labelling duration 1.73 seconds, post-labelling delay 0.75 seconds, repetition time (TR) $4000 \mathrm{~ms}$, echo time (TE) $34 \mathrm{~ms}, 45$ averages, $64 \times 64$ matrix, $240 \times 240 \mathrm{~mm}$ field of view (FOV), eight $6 \mathrm{~mm}$ thick slices with a $3 \mathrm{~mm}$ inter-slice gap covering a volume, with the axis aligned to the infracallosal line and extending inferiorly from just above the tentorium to the superior margin of the centrum semiovale. The slices were acquired in an interleaved, ascending order and the in plane resolution was $3.75 \times 3.75 \times 6 \mathrm{~mm}^{3}$. The labelling plane was positioned $4 \mathrm{~cm}$ inferior to the most caudal imaging slice. Double inversion labelling (with identical scanning parameters) was used for the control sequence, as this has no net effect on arterial water longitudinal magnetisation, but matches the magnetisation transfer effects in the labelled image. Control and labelled image collection was interleaved. The entire perfusion scan for each subject took 6 minutes, with a further 3 minutes required for the acquisition of a $T_{1}$ map using a 3 point inversion recovery technique in order to exactly match the distortions of the perfusion images (inversion time $=1$ second and 1.6 seconds, and one measure without inversion to calculate $\mathrm{M}_{0}$ (the equilibrium tissue magnetisation), TR 7.2 seconds).

All MS subjects had conventional spin echo proton density (PD) and $\mathrm{T}_{2}$ weighted images (TR $3000 \mathrm{~ms}$, TE 15/90 ms, matrix size $256 \times 256$; FOV $240 \times 240 \mathrm{~mm}$; 46 contiguous slices of $3 \mathrm{~mm}$ thickness) acquired prior to the CASL sequence. A two dimensional $\mathrm{T}_{1}$ weighted protocol (TR $620 \mathrm{~ms}$, TE $20 \mathrm{~ms}$, matrix size $256 \times 256$; 46 contiguous slices of $3 \mathrm{~mm}$ thickness) was also acquired. Total scanning time was about 35 minutes.

Quantitative perfusion maps were calculated from the CASL images and $\mathrm{T}_{1}$ map using a two compartment methodology described elsewhere. ${ }^{16}$ The model assumed the following parameters: inversion efficiency $0.7 ; \mathrm{T}_{1}$ of blood $1.4 \mathrm{~s}$; arrival time from the labelling plane to the imaging slab, $0.32-0.83 \mathrm{~s}$ (increasing with slice distance, assuming a linear path, with a blood velocity of $14 \mathrm{~cm} / \mathrm{s}$ ); permeability surface area product (PS) /blood water content $\left(\mathrm{V}_{\mathrm{bw}}\right)=1.5 /$ $0.05=30 /$ minute .

\section{Image processing}

Perfusion image spatial pre-processing

To allow regional quantification in WM and GM, the quantitative perfusion maps were spatially pre-processed using statistical parametric mapping (SPM '99, Wellcome Department of Cognitive Neurology, Institute of Neurology, London, UK). The perfusion images were spatially normalised into a standardised space defined by the EPI template (Montreal Neurological Institute) and subsequently smoothed with a $12 \mathrm{~mm}$ full width half maximum kernel. Extracerebral contributions were minimised using a whole brain mask and absolute lower limit perfusion threshold of $5 \mathrm{ml} / \mathrm{min} / 100 \mathrm{ml}$.

\section{Global evaluation of white matter perfusion}

In house software ${ }^{21}$ was used to register the $T_{1}$ map to the perfusion images. Segmentation of the perfusion maps was achieved using $T_{1}$ values derived from previous studies ${ }^{22-24}$ (500-800 ms for WM). This narrow limit was chosen to ensure high specificity for WM selection. Quantitative WM perfusion values for each slice were then averaged and weighted for the number of voxels to give an average WM perfusion value $(\mathrm{ml} / \mathrm{min} / 100 \mathrm{ml})$ for each subject. GM perfusion values were not used for this study because the $\mathrm{T}_{1}$ segmentation could not reliably avoid WM lesion contamination owing to similarities between GM and focal lesion $\mathrm{T}_{1}$ relaxation times. ${ }^{25}$

\section{Lesion quantification}

All images were displayed on a Sun workstation (Sun Microsystems, Mountain View, CA, USA) using the Dispimage display software package (Plummer, Department of Medical Physics, University College Hospitals NHS Trust, London, UK). Lesions were outlined using a semi-automated contouring technique ${ }^{26}$ on the $\mathrm{PD}$ weighted images. $\mathrm{T}_{1}$ weighted images were used to identify hypointense WM lesions using the same threshold method. These lesion segmentations were used to calculate brain $\mathrm{T}_{1}$ and $\mathrm{T}_{2}$ lesion loads.

\section{Statistical analysis}

For all statistical models employed, $\mathrm{p}<0.05$ was considered significant and in the analyses of perfusion maps all $p$ values were corrected for multiple comparisons. Individual MS subgroups were compared with all controls, with the potential effect of any age difference between the groups included and accounted for in each model.

Table 1 Entry stratification of all recruited subjects

\begin{tabular}{llrrlll}
\hline MS subtype & $\mathbf{n}$ & $\mathbf{M}$ & $\mathbf{F}$ & Mean age (range), years & Median EDSS (range) & $\begin{array}{l}\text { Median disease duration } \\
\text { (range), years }\end{array}$ \\
\hline Controls & 34 & 15 & 19 & $40.7(20-67)$ & 0 & - \\
All MS groups & 60 & 21 & 39 & $48.1(17-69)$ & $4.0(0-8.5)$ & $16(1-40)$ \\
Relapsing remitting & 21 & 8 & 13 & $38.9(17-59)$ & $2.5(0-6.5)$ & $7.0(0-6)$ \\
$\quad$ No treatment & 10 & 3 & 7 & $37.9(17-55)$ & $2.5(1-6.5)$ & $10(1-31)$ \\
$\quad$ On $\beta$-interferon & 11 & 5 & 6 & $39.8(27-59)$ & $6.0(2-8)$ & $18(7-40)$ \\
Secondary progressive & 14 & 3 & 11 & $51.2(30-65)$ & $6.5(3.5-8.5)$ & $16(8-34)$ \\
Primary progressive & 12 & 5 & 7 & $55.7(40-69)$ & $2.5(1-3)$ & $24(20-36)$ \\
Benign & 13 & 5 & 8 & $52.6(40-60)$ & \\
\hline
\end{tabular}


Statistical parametric mapping analysis of perfusion maps

SPM employs a general linear model with Gaussian field theory to make statistical inferences about regional effects from the normalised SPM perfusion maps. ${ }^{27}$ The statistical model included age, gender, and disease status as covariates of interest to correct for any differences between control and patient groups. The subjects were included as random effects, with each subject contributing one pre-processed perfusion image. The design matrix also included the global mean intensities as confounders, which ensured that the results reflected regionally specific differences in perfusion. Following model estimation, "T contrasts" were specified to test the statistical significance of particular linear combinations of parameter estimates. Voxelwise significance was determined by using Gaussian random field theory ${ }^{28}$ and the resulting statistical maps $\left(\mathrm{SPM}_{(\mathrm{t})}\right.$ maps) were corrected for multiple comparisons over the whole perfusion volume. Contrasts were specified to compare the control group with MS subgroups and different MS subgroups with each other. In order to avoid reporting areas of doubtful physiological relevance, only local maxima coordinates with a voxel cluster $>50$ were used.

White matter perfusion values using $T_{1}$ segmentation A further multiple linear regression model was created to evaluate WM perfusion values derived from $\mathrm{T}_{1}$ segmentation values. In the model, WM perfusion was the dependent variable, while gender and disease subgroup were included as categorical variables, and age as a continuous covariate to assess absolute disease effects allowing for age and gender.

\section{Correlations between perfusion measures and clinical measures}

To quantify the associations between perfusion and clinical status, models included age and gender as before, along with either MSFC as a continuous covariate or dichotomised EDSS values (mild $0-3$, moderate or severe $\geqslant 3.5$ ) as a categorical variable.

\section{RESULTS}

\section{Statistical parametric mapping based perfusion analysis}

Smoothed, normalised perfusion maps were generated for 33 controls and 59 MS subjects. Maps from one control and one secondary progressive subject were not included owing to inadequate normalisation to the EPI template.

The most prominent abnormalities were seen in primary and secondary progressive subgroups, where subjects exhibited regions of reduced perfusion that involved both cortical and deep GM structures and adjacent WM areas (table 2, fig 1) compared with controls. Smaller regions of decreased perfusion were seen in patients with benign disease, and no areas of reduced perfusion were seen in the relapsing remitting group.

Compared with controls, there was one small region of increased perfusion ( 69 voxels, $\mathrm{p}=0.001$ ) in the secondary progressive group in right frontal subcortical WM, and another small area of increased perfusion (78 voxels, $\mathrm{p}=0.003$ ) in 10 relapsing remitting patients not receiving $\beta$-interferon in a predominantly WM region adjacent to the left precentral and superior temporal gyrus.

\section{White matter perfusion results using segmentation based on $T_{1}$ value range}

Absolute WM perfusion values were compared in the subjects. Some WM lesions had $\mathrm{T}_{1}$ relaxation values of above $800 \mathrm{~ms}$, and the resulting WM masks will have excluded such lesions. Table 3 lists the results using this technique.
The mean values in healthy subjects compare well with other studies. ${ }^{29}$ A significant increase in WM perfusion was observed when all MS subjects were compared with controls $(\mathrm{p}=0.007)$. In the subgroup analysis, a significant WM perfusion increase was also seen in the pooled relapsing remitting cohort $(p=0.03)$ and secondary progressive subjects $(p=0.02)$ when compared with controls. The relapsing remitting group not on $\beta$-interferon showed a significant increase in perfusion $(p=0.04)$ whilst statistical significance was not reached in those on $\beta$-interferon $(p=0.14)$. There was weak evidence for increased WM perfusion in the benign MS group $(p=0.08)$, when compared with controls. No WM perfusion differences were detected between primary progressive subjects and controls. There were no statistically significant differences seen when comparing any of the MS subtypes directly.

\section{Correlations between perfusion measures and MRI lesion load or clinical measures}

In the SPM analysis there was no evidence of a correlation between perfusion measures and either lesion load or clinical outcomes. However in the $\mathrm{T}_{1}$ segmented WM perfusion analysis, there was evidence that correlations between PD lesion volumes and WM perfusion differed between MS subtypes $(p=0.03)$, with a suggestion that WM perfusion may be increasing more with PD lesion volume in the secondary progressive group than in other subtypes. There were no other significant correlations.

\section{DISCUSSION}

Previous studies of cerebral perfusion in MS have used PET or SPECT and have revealed areas of decreased perfusion in GM and WM. ${ }^{13-15}$ Exogenous contrast MR methods have been

Table 2 SPM analysis: regions of hypoperfusion (voxel clusters with significance of patient groups compared with controls) in the combined multiple sclerosis cohort and clinical subgroups of multiple sclerosis.

\begin{tabular}{|c|c|c|c|c|}
\hline Region & All MS & SP & PP & B \\
\hline $\begin{array}{l}\text { Deep grey matter } \\
\text { and adjacent white } \\
\text { matter }\end{array}$ & $\begin{array}{l}50 \% \text { GM; } \\
50 \% \text { WM }\end{array}$ & $\begin{array}{l}50 \% \text { GM; } \\
50 \% \text { WM }\end{array}$ & $\begin{array}{l}52 \% \text { GM; } \\
48 \% \text { WM }\end{array}$ & $\begin{array}{l}43 \% \text { GM; } \\
57 \% \text { WM }\end{array}$ \\
\hline $\begin{array}{l}\mathrm{R} \text { and } \mathrm{L} \text { thalamus, } \\
\text { caudate and } \\
\text { extranuclear areas }\end{array}$ & $\begin{array}{l}2399 \\
p \leqslant 0.001\end{array}$ & $\begin{array}{l}473 \\
p=0.001\end{array}$ & $\begin{array}{l}2214 \\
p \leqslant 0.001\end{array}$ & $\begin{array}{l}143 \\
p=0.004\end{array}$ \\
\hline $\begin{array}{l}\text { Cortical grey matter } \\
\text { and adjacent white } \\
\text { matter }\end{array}$ & $\begin{array}{l}66 \% \text { GM; } \\
34 \% \mathrm{~W}\end{array}$ & $\begin{array}{l}56 \% \text { GM; } \\
44 \% \mathrm{~W}\end{array}$ & $\begin{array}{l}56 \% \text { GM; } \\
44 \% \mathrm{~W}\end{array}$ & $\begin{array}{l}76 \% \text { GM; } \\
24 \% \mathrm{~W}\end{array}$ \\
\hline $\begin{array}{l}\mathrm{R} \text { and } \mathrm{L} \text { middle } \\
\text { frontal and } \\
\text { precentral and } \\
\text { postcentral gyri } \\
\text { and inferior parietal } \\
\text { areas }\end{array}$ & $\begin{array}{l}1450 \\
p \leqslant 0.001\end{array}$ & $\begin{array}{l}1469 \\
p \leqslant 0.001\end{array}$ & $\begin{array}{l}1802 \\
p \leqslant 0.001\end{array}$ & $\begin{array}{l}526 ; \\
p \leqslant 0.001\end{array}$ \\
\hline $\begin{array}{l}R \text { and } L \text { superior } \\
\text { frontal and medial } \\
\text { gyrus }\end{array}$ & $\begin{array}{l}1049 \\
p \leqslant 0.001\end{array}$ & $\begin{array}{l}614 ; \\
p \leqslant 0.001\end{array}$ & $\begin{array}{l}4668 \\
p \leqslant 0.001\end{array}$ & NS \\
\hline $\begin{array}{l}R \text { and } L \text { precuneus } \\
\text { and cingulate gyri }\end{array}$ & $\begin{array}{l}390 \\
p \leqslant 0.001\end{array}$ & NS & $\begin{array}{l}824 ; \\
p \leqslant 0.001\end{array}$ & NS \\
\hline $\begin{array}{l}R \text { and } L \text { paracentral } \\
\text { lobule }\end{array}$ & $\begin{array}{l}259 \\
p=0.001\end{array}$ & NS & NS & NS \\
\hline $\begin{array}{l}\text { L superior parietal } \\
\text { lobule and subgyral } \\
\text { areas }\end{array}$ & $p=0.007$ & NS & $\begin{array}{l}210 ; \\
p=0.010\end{array}$ & NS \\
\hline \multicolumn{5}{|c|}{$\begin{array}{l}\text { No regions of hypoperfusion were detected in the relapsing remitting } \\
\text { subgroup. } \\
\text { MS, multiple sclerosis; SP, secondary progressive; PP, primary } \\
\text { progressive; B, benign; GM, grey matter; WM, white matter; R, right; L, } \\
\text { left. } \\
\text { Percentages of grey and white matter for each subgroup are derived from } \\
\text { the total number of clusters in these regions as determined using the } \\
\text { Montreal Neurological Institute brain template. }\end{array}$} \\
\hline
\end{tabular}



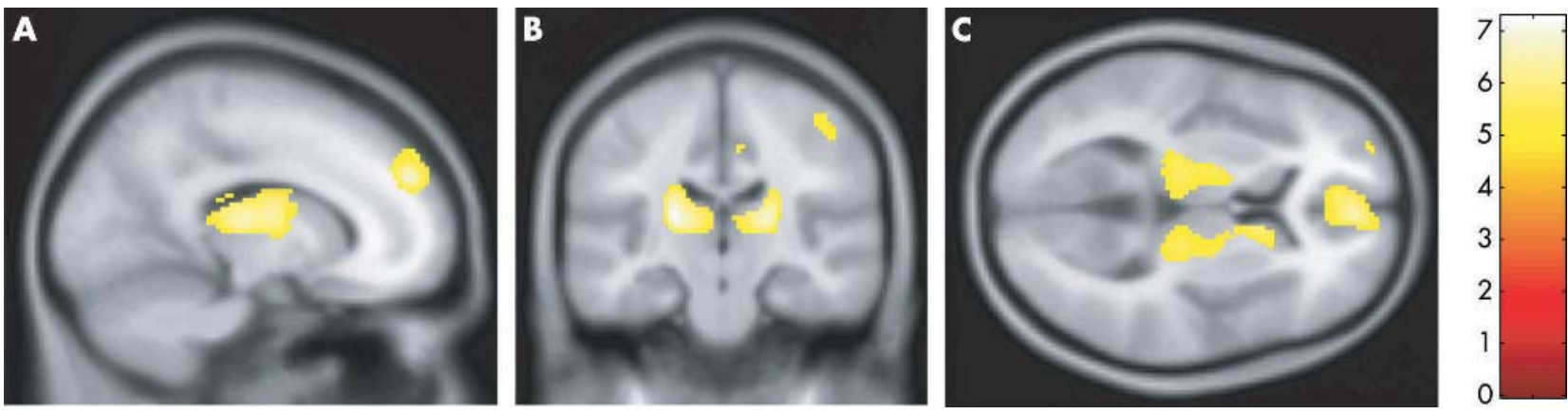

Figure 1 Regions of perfusion decrease in primary progressive subjects compared with normal controls. The colour bar indicates the T score.

used to evaluate cerebral blood volume in MS lesions with the largest of the studies showing, in 25 patients, an increase in cerebral blood volume in enhancing lesions and a decrease in $\mathrm{T}_{1}$ weighted hypointense lesions. ${ }^{30}$

The present study is the first to quantify cerebral perfusion in MS using a non-invasive method. The main findings in this study were a reduction of perfusion of GM structures, especially deep GM, and an increase in perfusion in WM.

\section{Decreased grey matter perfusion}

Using SPM, there was a large number of regions with lower perfusion located in both cortical and deep GM and adjacent WM regions. Neuronal and axonal dysfunction and loss in GM, with a corresponding reduction in local metabolic activity, may be the most likely causes of decreased GM perfusion. These findings are generally concordant with previous perfusion studies. ${ }^{13-15}$ Another possible explanation for non-homogeneity of perfusion could be a 'steal phenomenon' occurring in the local vascular network as a result of the redistribution of perfusion caused by abnormalities of brain activity in other regions. Additional, more localised, perfusion studies are required to investigate this further.

The primary progressive subjects exhibited the largest areas of reduced perfusion (table 2, fig 1 ), consistent with axonal loss being a prominent neuropathological feature in this subgroup. $^{31}$ Secondary progressive subjects also revealed substantial areas of reduced perfusion (table 2), which is again consistent with neuroaxonal loss or dysfunction observed using other putative MR markers of axonal integrity at this stage of disease. ${ }^{67}$ The analysis of data derived from subjects with benign MS showed only limited areas of decreased perfusion (table 2 ), which could reflect a generally less severe underlying pathology, mirroring the clinical phenotype. No regions of reduced perfusion were seen in the relapsing remitting group, which could be consistent with this subgroup having less neuroaxonal damage.

The evidence for hypoperfusion in MS patients relative to controls was apparent in both cortex and deep GM, and was especially striking in the thalamus and caudate nuclei. A recent MR study in MS has reported a decrease in thalamic volume and $\mathrm{N}$-acetyl aspartate. ${ }^{32}$ Furthermore, evaluations of glucose uptake in MS using PET have revealed areas of low metabolic function in the thalamus. ${ }^{33}{ }^{34}$ The decreased thalamic perfusion observed in our pooled MS cohort is consistent with neuronal loss or dysfunction in this structure. The decreased perfusion in cortex and deep GM structures may also be indicative of disconnection between cortical regions and subcortical relay systems due to demyelination. The observation of decreased perfusion in the primary and secondary progressive subgroups of MS suggests that this non-invasive imaging measure has the potential to investigate the adverse effects of neuroaxonal damage on brain metabolism in MS. In addition, as PET metabolic studies suggest a possible correlation between areas of low function and cognitive ${ }^{33}$ or memory disturbance, ${ }^{34}$ ASL may provide a potentially useful non-invasive evaluation into these symptoms in MS.

\section{Increased white matter perfusion}

Global assessment, using segmented $\mathrm{T}_{1}$ maps, revealed raised perfusion in MS WM compared with controls. Raised perfusion was evident only in relapsing remitting and secondary progressive subgroups, and in relapsing remitting subjects it was only apparent in those not being treated with $\beta$-interferon; these subgroup findings in global WM were supported by observations of small regions of increased WM perfusion using SPM.

The increase in WM perfusion may reflect higher metabolic activity due to an increase in cell number and activity in normal appearing WM or WM lesions (both normal appearing $\mathrm{WM}$ and lesions with a $\mathrm{T}_{1}$ relaxation time of less than $800 \mathrm{~ms}$ were included in the globally segmented WM). In normal appearing WM in MS, such features include astrocyte proliferation. ${ }^{35}$ In lesions, especially if of recent onset, inflammatory features including perivascular cuffs of lymphocytes and macrophage infiltrates may be prominent. ${ }^{1}$ Further evidence of the potential role of inflammation in WM in MS is provided by PET studies (using benzodiazepine agonists as a microglial marker) that show increased activity in gadolinium enhancing lesions and in normal appearing WM of subjects with greater disease progression. ${ }^{36}{ }^{37}$ In addition, further emphasising that perfusion may increase in areas of active inflammation in MS, a recent gadolinium bolus study demonstrated an increase in cerebral blood flow in areas of WM just before the area transformed into a gadolinium enhancing lesion. ${ }^{38}$

Although gadolinium enhanced scans were not obtained to investigate the presence of inflammatory lesions, it is known from previous studies that such lesions occur more often in relapsing remitting and secondary progressive MS than in primary progressive and benign $\mathrm{MS},{ }^{39} 40$ and are less common in patients receiving $\beta$-interferon. ${ }^{41}$ Our observation with the relapsing remitting subgroup not treated with $\beta$-interferon should, however, be interpreted cautiously, as there were only 10 subjects in this cohort and the increase was only marginally significant.

The analysis of WM perfusion using SPM suggests a complex pattern of regional perfusion. Although small foci of increased perfusion were observed in relapsing remitting and secondary progressive subgroups, areas of decreased perfusion were also seen, especially adjacent to the areas of decreased deep GM perfusion observed in patients with primary progressive or secondary progressive MS. Axonal loss has been described in WM lesions and normal appearing $\mathrm{WM}^{42}{ }^{43}$ and if extensive, might be expected to result in 
Table 3 White matter perfusion results $\left(\mathrm{ml} / \mathrm{min} / 100 \mathrm{ml}\right.$ ) based on $\mathrm{T}_{1}$ segmentation in all subjects

\begin{tabular}{|c|c|c|c|c|c|c|}
\hline Subject & $\mathbf{n}$ & $\begin{array}{l}\text { WM perfusion } \\
\mathrm{ml} / \mathrm{min} / 100 \mathrm{ml} \text { (SD) }\end{array}$ & $\begin{array}{l}\text { MS-control } \\
\text { difference* }\end{array}$ & $\mathbf{p}^{*}$ & $\begin{array}{l}\text { Median } T_{1} \text { lesion } \\
\text { volume (range), } \mathrm{ml}\end{array}$ & $\begin{array}{l}\text { Median PD } \\
\text { lesion volume (range), ml }\end{array}$ \\
\hline Controls & 34 & $24.2(4.2)$ & - & - & 0 & 0 \\
\hline All MS groups & 60 & $27.2(5.3)$ & 2.89 & 0.007 & $1.2(0-15.3)$ & $5.7(0.1-52.6)$ \\
\hline Relapsing remitting & 21 & $27.4(4.7)$ & 3.12 & 0.03 & $0.7(0-8.3)$ & $4.6(0.1-52.6)$ \\
\hline No treatment & 10 & $28.1(5.3)$ & 3.72 & 0.04 & $0.6(0-7.9)$ & $5.5(0.3-52.6)$ \\
\hline On $\beta$-interferon & 11 & $26.7(4.1)$ & 2.56 & 0.14 & $0.7(0-8.3)$ & $3.0(0.1-22.4)$ \\
\hline Secondary progressive & 14 & $28.3(5.7)$ & 3.72 & 0.02 & $2.8(0.2-15.3)$ & $10.3(1.3-36.8)$ \\
\hline Primary progressive & 12 & $25.8(5.0)$ & 1.62 & 0.33 & $1.7(0-10.4)$ & $10.1(1.3-44.9)$ \\
\hline Benign & 13 & $27.1(6.2)$ & 2.83 & 0.08 & $2.0(0.3-9.8)$ & $5.4(0.9-19.4)$ \\
\hline
\end{tabular}

regions with decreased metabolic activity and hence perfusion.

\section{Methodological considerations}

ASL relies upon a reduction in the tissue signal intensity associated with the passage of magnetically labelled blood through capillaries. Relative signal loss allows the quantification of perfusion. ${ }^{44}$ A new two compartment model was used to quantify perfusion. ${ }^{16}$ It includes a correction for the selective permeability of the blood brain barrier, producing a technique with greater physiological accuracy, as previous assumptions of free permeability of the capillary wall to water lead to an overestimation of perfusion, particularly in the WM where the loss of label (due to $\mathrm{T}_{1}$ relaxation) is approximately twice as fast as in blood. In addition, ASL is potentially more accurate in MS than using a gadolinium bolus technique, as the latter assumes that the label remains intravascular, and as such, perfusion values may be affected by blood-brain barrier breakdown, which is known to occur in $\mathrm{MS}^{45}$

With CASL and two compartment modelling, an increase in blood-brain barrier permeability to water in MS compared with controls could result in a small underestimation of perfusion, which could contribute to some of the perfusion reductions noted, but not the white matter increases. However, simulations of the two compartment model show that even relatively large changes in the permeability constant result in minimal changes to the perfusion measurements. ${ }^{16}$ Additionally, it would be difficult to model exactly for permeability differences between MS subjects and controls as this would vary not only between MS subgroups but also within subgroups, as it could be influenced by the inter-subject variability of inflammatory activity. Furthermore, disease related effects on $T_{1}$ relaxation values should not cause any significant error as these are measured and included in the calculation. A delay time between arterial water inversion and image acquisition is used to allow a high proportion of the tagged blood to reach its final tissue destination thereby minimising the contribution from blood transported in larger non-perfusing vessels. It is theoretically possible that a specific effect on the blood $\mathrm{T}_{1}$ relaxation time due to MS may significantly alter the measured perfusion result. However, there is a paucity of evidence to suggest that MS has such an effect, hence, this technique offers a potentially more accurate evaluation of perfusion in MS than previous approaches.

Further improvements, however, would be beneficial, particularly regarding improved focal lesion segmentation on the perfusion maps. The acquisition of a $\mathrm{T}_{2}$ weighted sequence that is fully registered with the perfusion images would be desirable in this respect. An increased number of signal averages to improve the signal to noise ratio would improve resolution but at the cost of increased scan time, and hence increase any potential subject motion artefact. Therefore, the optimal CASL sequence should balance these two competing influences. Image acquisition must be achieved within the time frame of the $T_{1}$ relaxation time of blood, necessitating high speed echoplanar imaging acquisition with relatively low resolution and limited brain coverage. Higher field strength scanners would increase the $T_{1}$ relaxation time and signal to noise ratio, although specific absorption rate limitations may occur above $1.5 \mathrm{~T}$.

\section{CONCLUSIONS}

This study of patients with MS has revealed reduced perfusion, especially in the GM, compared with controls. This was most apparent in the primary and secondary progressive subgroups. The decreases affected both cortical and deep GM structures, and are consistent with decreased neuronal metabolic activity or neuronal loss. Elevated perfusion was seen in WM in relapsing remitting and secondary progressive MS, possibly reflecting an increase in cell number and metabolic activity. Further investigation of the longitudinal changes in perfusion, and possible correlations with cognitive dysfunction and the effects of therapeutic interventions, particularly using methodological improvements such as higher field scanning, may provide further insights.

\section{ACKNOWLEDGEMENTS}

We thank D Alsop for the use of his CASL sequence, the Multiple Sclerosis Society of Great Britain and Northern Ireland for their continuing support of the NMR Research Unit (including W Rashid), the Brain Research Trust UK for support of L M Parkes, the Wellcome Trust for support of G T Ingle and M R Symms, Schering AG for support of D T Chard and The Mrs F B Laurence Trust and The Barnwood House Trust for support of A T Toosy. We are grateful to all the people who kindly agreed to take part in this study.

\section{Authors' affiliations}

W Rashid, G T Ingle, D T Chard, A T Toosy, D R Altmann, M R Symms, P S Tofts, A J Thompson, D H Miller, MS NMR Research Unit,

Departments of Neuroinflammation and Headache, Brain Injury and Rehabilitation, Institute of Neurology, University College London, UK L M Parkes, F.C. Donders Centre for Cognitive Neuroimaging, Trigon 181, NL-6500 HB Nijmegen, The Netherlands

D R Altmann, London School of Hygiene and Tropical Medicine, Keppel St, London, UK

M R Symms, MRI Unit, National Society of Epilepsy, Chalfont St. Peter, Buckinghamshire, UK

Competing interests: none declared

\section{REFERENCES}

1 Lassmann H. Neuropathology in multiple sclerosis: new concepts. Mult Scler 1998;4:93-8.

2 Lassmann H, Suchanek G, Ozawa K. Histopathology and the blood cerebrospinal fluid barrier in multiple sclerosis. Ann Neurol 1994;36(Suppl):42-6.

3 Losseff NA, Wang L, Lai HM, et al. Progressive cerebral atrophy in multiple sclerosis. A serial MRI study. Brain 1996;119:2009-19.

4 De Stefano N, lannucci G, Sormani MP, et al. MR correlates of cerebral atrophy in patients with multiple sclerosis. J Neurol 2002;249:1072-7. 
5 Arnold DL, Matthews PM, Francis G, et al. Proton magnetic resonance spectroscopy of human brain in vivo in the evolution of multiple sclerosis: assessment of the load of disease. Magn Reson Med 1990;14:154-9.

6 Matthews PM, Pioro E, Narayanan S, et al. Assessment of lesion pathology in multiple sclerosis using quantitative MRI morphometry and magnetic resonance spectroscopy. Brain 1996;1 19:715-22.

7 Fu L, Matthews PM, De Stefano N, et al. Imaging axonal damage of normal appearing white matter in multiple sclerosis. Brain 1998;121:103-13.

8 Trapp BD, Ransohoff R, Rudick R. Axonal pathology in multiple sclerosis: relationship to neurologic disability. Curr Opin Neurol 1999;12:295-302.

9 Keir SL, Wardlaw JM. Systematic review of diffusion and perfusion imaging in acute ischaemic stroke. Stroke 2002;31:2723-31.

10 Lojkowska W, Ryglewicz D, Jedrezejczak T, et al. SPECT as a diagnostic test in the investigation of dementia. J Neurol Sci 2002;203-204:215-19.

11 Varrone A, Pappata S, Caraco C, et al. Voxel-based comparison of rCBF SPET images in frontotemporal dementia and Alzheimer's disease highlights the involvement of different cortical networks. Eur J Nucl Med Mol Imaging 2002;29:1447-54

12 Pasquier J, Michel BF, Brenot-Rossi I, et al. Value of (99m) Tc-ECD SPET for the diagnosis of dementia with Lewy bodies. Eur J Nucl Med Mol Imaging 2002;29:1342-8.

13 Brooks DJ, Leenders KL, Head G, et al. Studies on regional cerebral oxygen utilisation and cognitive function in multiple sclerosis. J Neurol Neurosurg Psychiatry 1984;47:1182-91.

14 Lycke J, Wikkelso C, Bergh AC, et al. Regional cerebral blood flow in multiple sclerosis measured by single photon emission tomography with technetium$99 \mathrm{~m}$ hexamethylpropyleneamine oxime. Eur Neurol 1993;33:163-7.

15 Pozzilli C, Passafiume D, Bernardi S, et al. SPECT, MRI and cognitive functions in multiple sclerosis. J Neurol Neurosurg Psychiatry 1991;54:110-15.

16 Parkes LM, Tofts PS. Improved accuracy of human cerebral blood perfusion measurements using arterial spin labeling: accounting for capillary water permeability. Magn Reson Med 2002;48:27-41

17 Lublin FD, Reingold SC. Defining the clinical course of multiple sclerosis: results of an international survey. National Multiple Sclerosis Society (USA) Advisory Committee on Clinical Trials of New Agents in Multiple Sclerosis. Neurology 1996;46:907-11

18 Poser CM, Paty DW, Scheinberg L, et al. New diagnostic criteria for multiple sclerosis: guidelines for research protocols. Ann Neurol 1983;13:227-31.

19 Kurtzke JF. Rating neurologic impairment in multiple sclerosis: an expanded disability status scale (EDSS). Neurology 1983;33:1444-52.

20 Fisher JS, Kak AJ, Kniker JE, et al. Administration and scoring manual for the multiple sclerosis functional composite measure (MSFC). New York: Demes, 1999.

21 Symms MR, Barker GJ, Holmes P, et al. A rapid automated system for detection of serial changes in transient ischaemic attack using registration and subtraction of three-dimensional images. Proc Intl Soc Mag Reson Med 1997; 1:584.

22 Alsop DC, Detre JA. Reduced transit-time sensitivity in noninvasive magnetic resonance imaging of human cerebral blood flow. J Cereb Blood Flow Metab 1996;16:1236-49.

23 Steen RG, Gronemeyer SA, Kingsley PB, et al. Precise and accurate measurement of proton $\mathrm{T} 1$ in human brain in vivo: validation and preliminary clinical application. J Magn Reson Imaging 1994;4:681-91.

24 Vymazal J, Righini A, Brooks RA, et al. T1 and T2 in the brain of healthy subjects, patients with Parkinson disease, and patients with multiple system atrophy: relation to iron content. Radiology 1999;211:489-95.
25 Miller DH, Johnson G, Tofts PS, et al. Precise relaxation time measurements of normal-appearing white matter in inflammatory central nervous system disease. Magn Reson Med 1989;11:331-6.

26 Filippi M, Gawne-Cain ML, Gasperini C, et al. Effect of training and different measurement strategies on the reproducibility of brain MRI lesion load measurements in multiple sclerosis. Neurology 1998;50:238-44.

27 Friston KJ, Holmes AP, Worsley KJ, et al. Statistical parametric maps in functional imaging: A general linear approach. Hum Brain Mapp 1995:2:189-210.

28 Worsley KJ, Marrett S, Neelin P, et al. A unified statistical approach for determining images of cerebral activation. Hum Brain Mapp 1996;4:58-73.

29 Leenders KL, Perani D, Lammertsma AA, et al. Cerebral blood flow, blood volume and oxygen utilization. Normal values and effect of age. Brain 1990;113:27-47.

30 Haselhorst R, Kappos L, Bilecen D, et al. Dynamic susceptibility contrast MR imaging of plaque development in multiple sclerosis: application of an extended blood-brain barrier leakage correction. J Magn Reson Imaging 2000; 11:495-505.

31 Bruck W, Lucchinetti $\mathrm{C}$, Lassmann $\mathrm{H}$. The pathology of primary progressive multiple sclerosis. Mult Scler 2002;8:93-7.

32 Cifelli A, Arridge $M$, Jezzard P, et al. Thalamic neurodegeneration in multiple sclerosis. Ann Neurol 2002;52:650-3.

33 Blinkenberg $M$, Rune $K$, Jenson CV, et al. Cortical cerebral metabolism correlates with MRI lesion load and cognitive dysfunction in MS. Neurology 2000;54:558-64.

34 Paulesu $E$, Perani $D$, Fazio $F$, et al. Functional basis of memory impairment in multiple sclerosis: a [18F] FDG PET study. Neuroimage 1996;4:87-96.

35 Allen IV, Glover G, Anderson R. Abnormalities in the macroscopically normal white matter in cases of mild or spinal multiple sclerosis (MS). Acta Neuropathol Suppl (Berl) 1981;7:176-8.

36 Debruyne JC, Versijpt J, Van Laere KJ, et al. PET visualisation of microglia in multiple sclerosis patients using $\left[{ }^{11} \mathrm{C}\right] \mathrm{PK} 11195$. Eur J Neurol 2003; 10:257-64.

37 Banati RB, Newcombe J, Gunn RN, et al. The peripheral benzodiazepine binding site in the brain in multiple sclerosis. Quantitative in vivo imaging of microglia as a measure of disease activity. Brain 2000;123:2321-37.

38 Wuerfel J, Bellmann-Strobl J, Brunecker P, et al. Changes in cerebral perfusion precede plaque formation in multiple sclerosis. Mult Scler 2003;9(Supp 1):102.

39 Thompson AJ, Kermode AG, Wicks D, et al. Major differences in the dynamics of primary and secondary progressive multiple sclerosis. Ann Neurol 1991:29:53-62.

40 Thompson AJ, Miller D, Youl B, et al. Serial gadolinium-enhanced MRI in relapsing-remitting multiple sclerosis of varying disease duration. Neurology 1992;42:60-3.

41 Li DK, Paty DW. Magnetic resonance imaging results of the PRISMS trial: a randomised, double-blind, placebo-controlled study of interferon-beta la in relapsing-remitting multiple sclerosis. Ann Neurol 1999;46:197-206.

42 Trapp BD, Peterson J, Ransohoff RM, et al. Axonal transection in lesions of multiple sclerosis. N Engl J Med 1998;338:278-85.

43 Evangelou N, Esiri MM, Smith S, et al. Quantitative pathological evidence for axonal loss in normal appearing white matter in multiple sclerosis. Ann Neurol 2000;47:391-5.

44 Detre JA, Leigh JS, Williams DS, et al. Perfusion imaging. Magn Reson Med 1992;23:37-45.

45 Tofts PS, Kermode AG. Blood brain barrier permeability in multiple sclerosis using labelled DTPA with PET, CT and MRI. J Neurol Neurosurg Psychiatry 1989;52:1019-20. 